\title{
Research on Customer Gender Oriented Packaging Design of Beverage Products
}

\author{
Tian $\mathrm{Su}$ \\ Henan Institute of Engineering \\ Zhengzhou, China 451191
}

\begin{abstract}
Beverage packaging design shall be positioned according to consumers' gender with market segment, give the suitable products to the right people, so as to better cater to different consumers' psychology. In this era of global culture and multimedia, personality is often closely related to people's lives. Only in constant development can innovation bring about the unprecedented shock.
\end{abstract}

Keywords-beverage packaging; gender; differentiation; personalization

\section{INTRODUCTION}

The packaging must be reflected by a certain material, shape and structure "while packaging design refers to the appearance design of various kinds of packaging ".

Packaging is indispensable in today's era under the condition of highly developed science and technology. Its main purpose is to protect the goods, facilitate circulation and consumption, promote sales, and satisfy people's material and spiritual needs. This will need to consider to protect the inside container to avoid damage and usage of the beverage, pay attention to beautiful appearance, with good visual appeal, fashion sense, to reflect the texture and grade of the goods.

With the change of the times, business competition is more and more intense, particularly in the beverage industry, and the wide varieties of drinks emerge endlessly. Consumers' purchase decision is made in front of the shelves in most cases. As a result, the role of packaging in influencing consumers' purchase decision is getting more and more attention. In today's beverage consumption market, carbonated drinks, juice, water, beer and alcoholic beverages each occupies different market due to consumers' different tastes. In addition, new varieties of drinks are released to the market one after another, such as multi-functional drinks, iced tea, mixed drinks, and they have taken part of the market from some famous beverages. The current beverage packaging must enhance the image of beverage, and transfer the information of the brand. In this era of global culture and multimedia, personality is often closely related to people's lives. Only by innovating in its constant development can bring people the unprecedented shock.

Today's beverage packaging design is not just for the sake of protection products in circulation, convenient transportation and promoting sales, it also carries a lot of information. As a container of products, beverage packaging has certain artistic charm in addition to its usefulness. This charm lies in deepening consumers' impression through the container's expression methods such as color, shape and texture, transferring product information to consumers, to arouse consumers' purchase desire. It has the value of continuous use due to this characteristic of packaging container. Nowadays, the market has entered into a personalized consumption and consumer psychology has been quite mature, beverage packaging design will tend to be more personalized. Beverage packaging design tended to be more and more targeted, because people of different gender like different tastes, so that great differences appear in the packaging of products.

If the consumption objects are positioned as all customers, consumers' satisfaction is certainly to be reduced. The product packing is unlikely to meet all the requirements of customers, resulting in consumers' lack of loyalty to the brand. We all know there are differences between brand satisfaction and brand loyalty, and there must be targeted market segments to hold firm the customers, and adopt different advertising sales approach against people of different consumption levels, to give the suitable products to the right person. Only the continued satisfaction can make the real loyalty, or the "right person" can't grow into the customer with firm relationship. The author thinks that beverage packaging design shall be positioned accurately according to gender of the customers, so as to better cater to consumer psychology, seize the consumer's desire, and meet different people's preferences and requirements.

\section{GENDER-BASED BEVERAGE MARKET SEGMENTATION}

Marketing master Kotler has pointed out that the center of modern strategic marketing can be defined as STP marketing, namely segmentation, targeting and positioning. All corporate marketing strategies must be carried out based on market segmentation. Enterprises cannot lock their market without segmentation during operation, and they will unable to find their positioning in market competition. Only with market segmentation, can the marketing strategy be differentiated. Therefore, market segmentation is an important part and platform of enterprise strategic marketing. The variable of market segmentation is various, many different methods of segmentation have been very popular, but these variables can be divided into four categories, namely: geographic region segmentation, demographic segmentation, psychological segmentation and behavior subdivision. 
At present, the domestic market segmentation of beverage manufacturers is mainly vertical, ranging from children's drinks to adult drinks; there are the single variety of beverages divided according to their ingredients, for example the mixed juice beverage such as orange juice, apple juice. If we jump out of the way of thinking and divide it according to gender, creatively put forward the simple and new segmentation method of "gender-based beverage", it will overturn the previous beverage market positioning method. This segmentation method is unexpected but reasonable.

\section{GENDER-BASEd BEVERAGe PROduct DESIGN}

The product's positioning design is the product of modern market economy. With the rapid development of science and technology, human's ability of producing products is greatly improved, and the differences between products are narrowed, similar products can be found everywhere. At this moment, it is impossible to gain consumers' cognition through the product's own characteristics, and consumer psychology also rises from a mere material field to the spiritual domain.

Product design shall meet the needs of society, and to constantly find and excavate the demand of consumers. This is the most important one in integrated marketing communication "4C" theory - the consumer demand. Along with the social progress and the development of economy and culture, people have more and more spiritual and psychological demand while the material needs are increasingly satisfied, and the demand is becoming more and more personalized. In the busy modern pace of life, men and women are under different pressure due to their different roles in society. Men want to have energy and vitality, to be more mature and self-confident; woman want to reduce their signs of aging, to have bright and clean skin as well as beautiful appearance, and to be more beautiful and confident. Under the increasing pressure, people are longing for the more sweet and romantic feelings.

\section{Gender-BASEd BeVERAge PaCKAgING DESIGN}

Packaging is the most direct part for the communication between the products and consumer, it is also a kind of carrier which can intuitively pass on the products' properties to consumers, reflecting the products' individuation and differentiation, extending the connotation and taste of the products, to attract consumers for resonance. Product' design elements, namely the visual elements such as modeling, color and text, give people different implication and association. For example, people can know the properties of the goods within the container directly through the size, shape and texture of the bottle, and understand different bias of the same product. The more typical one is perfume, as it can show the gender differences with different modeling and color.

One of the important design elements influencing beverage packaging design is the modeling of packaging, which is different from the principles of other architecture design and industrial product design. The shape of packaging design and container modeling design is determined by its own appealing objects. Plane has the feeling of level, smooth and concise; curved surface has the feeling of soft and gentle and full of elasticity; the round one is pure, plump, and square is rigorous, grave...which are the elements need to be considered when we research the gender-based beverage packaging design.

As the product visual element, text holds the extremely important position in packaging design. Text not only plays the important role of carrying and conveying a variety of text information, but its own visual image is an important kind of adornment and media. All kinds of product packaging text can have completely different image and style. For example, there is considerable difference of the text of cosmetic products for different genders, as the text on male cosmetics packaging often is often strong and tough with the feeling of high resilience; and the text of female cosmetic packaging is fine and beautiful with the feeling of graceful. In terms of the choice of color, people of different identity, age and culture have different color preferences. Red is a color with the aesthetic tendency of the Chinese nation, and red is usually used for women in terms of color matching for personal items especially common items. Generally, there are the several reasons for women' preference to red: first, red can foil a woman's skin more white, ruddy and charming; second, red represents enthusiasm, which is a kind of perceptual color, corresponding to women's inner beauty and the advantage of perceptual thinking with resonance; third, the psychological suggestion function of red is a "killer" of women, for instance, it is easy to make people associate with the delicate and charming flowers, beautiful spring scenery and romantic sentimental life with red. As for the outer appearance style, women often adopt the plump curve shape, focusing on the overall shape with fairing and turning processing; as for local details, the soft graphics or decoration inlaid are adopted. To convey women' enchanting and charming temperament, the means such as transparent material, soft material, mounted gems, and specular surface processing will be adopted, to convey the idea of female's delicate features.

For example, wine is favored by many female consumers because of its special effect of beauty and health. According to a survey on wine market made by the American council, 60\% of the high-end wine buyers are women, revealing the important role of women in wine consumption. Therefore, some packaging with women thinking as the leading factor can be designed, to highlight female characteristic in terms of outer packaging color, and add some ornaments as appropriate, which plays the role of "making the finishing point". Blue and silver are mainly adopted for men. Usually deep blue is adopted. Blue is the color of rational, with a sense of science and technology and machinery, which is suitable for men's temperament of strong and rational. As for the outward appearance, in order to emphasize men's wise, calm, generous and easy, such methods as metal, electroplating and leather surface treatment will be adopted. The relatively strong straight line is adopted for the line style, and the transition processing between the surfaces adopts the hard link, revealing its angular feature. For example, the energy drink that is popular in the market in recent years-Mizone, its mainly blue, people can feel its movement, strength, rational and masculinity through the design of blue bottle with angular line, which has met men's aesthetic standards, hold the characteristic of the consumer, and has won men's psychological needs. 
In the fierce market competition, gender has become an indicator of market segmentation. Many items which need not be differentiated based on gender shall be marked with labels for men and women in order to adapt to the demand of men's and women's personality, living habit and physiological characteristics. To design different packaging for the same kind of product for different gender has become the necessary marketing tool. The beverage packaging design with gender differentiation has grasped the public's consumption psychology, caused the emotional resonance, which is easy to be recognized, so as to stimulate mass consumption, and it is an approach to creative consumption boom with creative design. In today's increasingly competitive beverage market, to gain a foothold, consumer's psychology shall be seized, to conduct market segmentation for beverage products, enabling different people to find the beverage products suitable for themselves. The future beverage packaging design will need the differentiation, to manufacture the product packaging with differentiation, so that our products can be outstanding among numerous competitors.

\section{REFERENCES}

[1] Zhang Zhongyi, Zhang Libo. Discussion on Application of Color in Beverage Packaging [J]. Textile Industry and Technology, 2011, 06:5759.

[2] Wang Shaoshu. Influence of Female Consumer Demand on Household Products Packaging Design [J].Design, 2013,06:114-115.

[3] Li Jialong, Ouyang Chaoying. Study on Positioning and Design of Decorative Surface of Beverage Packaging [J]. Packaging Engineering, 2013,20:11-15.

[4] Shi Jiangbin, Liao Fengfeng, Wei Xia. Innovative Design of Beverage Packaging under Perceptual Consumption $[\mathrm{J}]$. Packaging Engineering, 2007,11:131-133+153.

[5] Liu Fei. Study on Creativity and Design of Individualized Packaging [D]. Shanghai Normal University, 2015. 